\title{
Screening of new British thraustochytrids isolates for docosahexaenoic acid (DHA) production
}

\author{
Loris Fossier Marchan ${ }^{1} \cdot$ Kim J. Lee Chang ${ }^{2} \cdot$ Peter D. Nichols ${ }^{2} \cdot$ Jane L. Polglase $^{3}$ • \\ Wilfrid J. Mitchell ${ }^{4}$ - Tony Gutierrez ${ }^{1}$
}

Received: 4 November 2016 / Revised: 16 April 2017 / Accepted: 17 April 2017 / Published online: 30 April 2017

(C) The Author(s) 2017. This article is an open access publication

\begin{abstract}
Thraustochytrids isolated from hot tropical and sub-tropical waters have been well studied for DHA and biodiesel production in the last decades. However, little research has been performed on the oils of cold water thraustochytrids, in particular from the North Sea region. In this study, thraustochytrid strains from British waters showed high relative levels of omega-3 long-chain $\left(\geq \mathrm{C}_{20}\right)$ polyunsaturated fatty acids (LC-PUFA), including docosahexaenoic acid (DHA, 22:6w3). The relative levels of DHA (as \% of total fatty acids, TFA) in the different British strains are hitherto amongst the highest recorded from any thraustochytrid screening study, with strain TL18 reaching up to 67\% DHA in modified Glucose-Yeast Extract-Peptone (GYP) medium. At this screening stage, low final biomass and fatty acid yield were observed in modified GYP and MarChiquita-Brain Heart Broth (MCBHB), while PUFA profiles (as \% of PUFA) remained unaltered regardless of the culture medium used. Hence, optimizing the medium and culture conditions to improve growth and lipid content, without impacting the relative percentage of DHA, has the potential to increase the final
\end{abstract}

Tony Gutierrez

tony.gutierrez@hw.ac.uk

1 Institute of Mechanical, Process \& Energy Engineering, School of Engineering and Physical Sciences, Heriot-Watt University, Edinburgh EH14 4AS, UK

2 CSIRO Oceans and Atmosphere, GPO Box 1538, Hobart, TAS 7001, Australia

3 Institute of Life and Earth Sciences, School of Energy, Geoscience, Infrastructure and Society, Heriot-Watt University, Edinburgh EH14 4AS, UK

4 Institute of Biological Chemistry, Biophysics and Bioengineering, School of Engineering and Physical Sciences, Heriot-Watt University, Edinburgh EH14 4AS, UK
DHA concentration. With this in mind, three strains were identified as promising organisms for the production of DHA. In the context of possible future industrial exploitation involving a winterization step, we investigated the recycling of the residual oil for biodiesel use. To do this, a mathematical model was used to assess the intrinsic properties of the byproduct oil. The results showed the feasibility of producing primary DHA-rich oil, assuming optimized conditions, while using the by-product oil for biodiesel use.

Keywords Thraustochytrid · Docosahexaenoic acid · Omega-3 fatty acids $\cdot$ Biodiesel $\cdot$ By-product

\section{Introduction}

Thraustochytrids are heterotrophic heterokonts or stramenopiles, certain strains of which are recognized for their ability to produce substantial amounts of omega-3 fatty acids, a high percentage of which is docosahexaenoic acid (DHA) (Lewis et al. 1999; Lee Chang et al. 2014). DHA is a major structural lipid which can be found as a constituent of phospholipids or triacylglycerols, or as free fatty acids in animals. It also has a key role in cell signalling, cell interaction and membrane fluidity (Colomer et al. 2007). DHA plays an important role in human health, in particular during the early stage of foetal and post-natal neuronal, retinal and immune system development (Swanson et al. 2012). There are positive correlations between DHA consumption and reduced disease, particularly in the prevention of cancers and cardiovascular diseases, improvement of inflammation response systems and maintenance of brain and learning functions (Horrocks and Yeo 1999; Lee Chang et al. 2013b). This knowledge has led to a growing demand for omega-3 supplemented food and other products. In fish, DHA has also been reported as 
essential for the normal growth and development of the animal, thus making it an indispensable component in aquaculture feeds (Sargent et al. 1999).

Currently, the production of omega-3-rich oils for human and animal diets relies primarily on wild-harvested fish and other marine animals such as krill (Pike and Jackson 2010). Paradoxically, this makes the expansion of the aquaculture industry directly limited by the global fishing capacity and its supply of DHA. However, with global fishing capacity reaching maximum levels since the early 1990s, aquaculture has overtaken the traditional wild-harvest fishing industry to meet the demand for fish and has become the fastest growing animal food-producing sector (FAO 2014). Hence, the supply of wild fish, and therefore omega- 3 oils, is becoming tighter, emphasizing the need for alternate, renewable and sustainable sources of DHA (Pike and Jackson 2010). To meet this demand, the potential of thraustochytrids as DHA producers has been recognized (Barclay et al. 1994), and some examples of the omega-3 oils they produce have since been developed into commercially available bio-ingredients, such as Life's DHA from DSM and DHAid from Lonza (Ratledge 2012). Nevertheless, further work is still needed to improve DHA yields from thraustochytrids, including the isolation and screening of new strains, together with their optimization using established or new fermentation strategies for production and the investigation of new substrates for even more cost-effective thraustochytrid growth.

In addition to the growing volume of research and development on the use of thraustochytrid oil in human and animal nutrition, there is also interest in the potential of these organisms for producing biodiesel (Lee Chang et al. 2012). With global economies depending almost entirely on fossil fuel resources, and taking into account the global depletion of natural reserves and the inextricable link between fossil fuel use and global warming, there is a need for sustainable alternatives to fossil fuel (Tietenberg and Lewis 2009). As a result, biodiesel has been promoted as an alternative to diesel fuel (European Commission 2003; Energy Policy Act 2005), and regulations for standardization of biodiesel were introduced, such as European standard EN 14214 and the ASTM D6751 in the USA and Canada. Since field crop-based biodiesel is associated with major socio-economic and environmental limitations, research focusing on single-cell oil-based biodiesel, including from thraustochytrids, has expanded (Meng et al. 2009). A major limitation to the implementation of thraustochytrid-derived oil is that the production of fish- or crop-derived oils remains more cost-effective, as a shortterm application.

Most of the research on DHA from thraustochytrids to date has been performed with sub-tropical strains, many of which have been isolated from Malaysian, Japanese and Chinese coastal waters (Fan et al. 2002; Li et al. 2009; Byreddy et al. 2015; Chen et al. 2015; Manikan et al. 2015). Other work has been done on isolates from temperate regions such as southern Australian waters and cold temperate waters of Canadian littoral (Burja et al. 2006; Lee Chang et al. 2012). Here, we present the first detailed investigation on the oil composition and content of several new thraustochytrid strains isolated from Scottish waters. We investigated the DHA content and the qualitative properties of fatty acid profiles of these strains, with a view for potential applications in animal and human nutrition. We also performed a mathematical analysis based on the assumption that a winterization or other separation/ fractionation step could be used for the purification of the DHA-rich oil, in order to investigate the potential use of the remaining by-product oil for biodiesel application.

\section{Materials and methods}

\section{Thraustochytrid strains, maintenance and inoculum preparation}

The thraustochytrid strains used in the present study are part of a unique British collection of strains isolated from lesser octopuses (Eledone cirrhosa), with a fatal ulcerative dermal necrosis at GPS coordinates $56^{\circ} 10^{\prime} \mathrm{N} 2^{\circ} 45^{\prime} \mathrm{W}$, from within the lairs in which the octopuses lived, and from rainbow trout (Oncorhynchus mykiss), at GPS coordinates $56^{\circ} 26^{\prime} \mathrm{N} 5^{\circ} 50^{\prime}$ W, also with skin lesions (Polglase 1980, 1981). Briefly, flasks containing $20 \mathrm{~mL}$ of sterile seawater were baited with sterile pine (Pinus montana) pollen. Flasks were then inoculated with a swab from a skin lesion or with $0.5 \mathrm{~mL}$ of water taken from the octopus lair. Thraustochytrid growth on the pollen grains was examined after 7, 10, 14, 21 and 28 days, and colonized pollen grains were used to inoculate agar plates (2\% pre-soaked Fadenagar in seawater, $0.2 \%$ bacteriological agar (Difco, Germany), $0.03 \%$ peptone, $0.003 \%$ yeast extract (Difco, Germany) and $0.3 \%$ malt extract (Biomaltz, Germany). The plates also contained penicillin (50 to $\left.100 \mathrm{IU} \mathrm{mL}^{-1}\right)$ and streptomycin $\left(100 \mu \mathrm{g} \mathrm{mL}^{-1}\right)$. From these, small colonies were taken to inoculate further pollen-baited flasks. A glass micro-loop was then used to separate a pollen grain bearing a single thraustochytrid, which was used to establish an initial pure culture in a seawater and pine pollen culture flask.

Flask cultures were kept at 7,10 or $15^{\circ} \mathrm{C}$ and sub-cultured approximately every 6 months until the beginning of the present study, when colonized pollen grains were plated on MCBHB agar (Rosa et al. 2011) and single colonies were isolated and transferred into MCBHB medium in flask culture. One further strain (SM01) from soil in a salt marsh $\left(56^{\circ} 02^{\prime} \mathrm{N}\right.$ $2^{\circ} 58^{\prime} \mathrm{W}$ ) was isolated similarly on pine pollen grains and MC agar (Rosa et al. 2011) in 2015 and subsequently maintained in MCBHB culture medium. The strain Sicyoidochytrium sp. NBRC 102979, obtained from the National Biological 
Resource Centre (NBRC) culture collection of the National Institute of Technology and Evaluation (NITE, Japan), was revived in $\mathrm{H}$ agar and medium (Honda et al. 1998) in halfstrength seawater, before being treated and maintained in the same condition as the British isolates on MCBHB. This strain, isolated from Japanese sub-tropical waters, was selected to compare its performance with isolates from cold northern European waters. Inocula were prepared from axenic cultures maintained in MCBHB, with $1 \mathrm{~mL}$ of culture transferred to $20 \mathrm{~mL}(5 \% v / v)$ MCBHB or modified GYP (depending on the type of medium to be used for lipid analysis) in static pharmacological glass culture flasks and incubated at $20{ }^{\circ} \mathrm{C}$ for 2 weeks.

\section{Culture conditions for lipid analysis}

The fatty acid profiles for each strain were evaluated in MCBHB, as previously described by Rosa et al. (2011), as well as using a modified version of the GYP media of Lee Chang et al. (2013a) (2\% glucose, $0.2 \%$ peptone, $0.5 \%$ yeast extract, $0.2 \%$ monosodium glutamate, $0.1 \%$ corn steep liquor and $1 \mathrm{~mL}$ of each metal solution and vitamin solution as defined by the authors). Artificial sea salts used in both of these media were replaced by a 1:1 mixture of natural seawater/ distilled water. Each strain was inoculated at $3 \%(v / v)$ into six 250-mL conical flasks for each medium and incubated on a rotary shaker $\left(21{ }^{\circ} \mathrm{C}, 100 \mathrm{rpm}\right)$. Optical density was measured at $600 \mathrm{~nm}$ using a Genesys 20 spectrophotometer (ThermoScientific, UK). At the end of exponential growth phase, three of the six flasks were terminated; the remaining three flasks were incubated for a further 4 days to reach late stationary phase. Cells were centrifuged $(2600 \times g$ for $10 \mathrm{~min})$ in $50-\mathrm{mL}$ Falcon tubes and washed twice with $20 \mathrm{~mL}$ of $0.01 \mathrm{M}$ phosphate buffered saline (PBS) at $\mathrm{pH}$ 7.2. Cell pellets were stored at $-20{ }^{\circ} \mathrm{C}$ and then freeze-dried for subsequent fatty acid analyses.

\section{Direct transesterification of biomass for fatty acid methyl ester production}

Freeze-dried cell pellets were directly transesterified to produce fatty acid methyl esters (FAME) as described by Lee Chang et al. (2011). Freeze-dried pellets were transferred into tared capped Pyrex test tubes and weighed. To each tube, $3 \mathrm{~mL}$ of methanol/chloroform/hydrochloric acid (10:1:1, $v / v /$ v) was added, and then the tubes were heated to $80^{\circ} \mathrm{C}$ in a water bath for $1 \mathrm{~h}$. After cooling to room temperature, $1 \mathrm{~mL}$ of MilliQ water was added, and the mixture was extracted by the addition of $2 \mathrm{~mL}$ of hexane/chloroform (4:1). After vortexing and centrifugation at $400 \times g$ for $5 \mathrm{~min}$, the upper non-aqueous layer of each sample was transferred to a clean test tube using a glass Pasteur pipette. Each mixture was extracted three times, and the combined extracted phases were then concentrated by blowing down under a stream of nitrogen gas until the volume was approximately $1 \mathrm{~mL}$. The mixture of FAME was then transferred to a $2-\mathrm{mL}$ amber glass vial (Agilent Technologies, UK) for subsequent fatty acid analysis.

\section{Determination of fatty acids profile}

FAME were analysed using gas chromatography (GC) to determine fatty acid composition, as described by Lee Chang et al. (2014). For this, samples were blown down under nitrogen gas to remove residual solvent. A known volume of dichloromethane containing the internal injection standard (19:0 FAME) was then added to each sample vial. GC was performed on an Agilent Technologies 7890A GC (USA) equipped with a non-polar Equity-1 fused silica capillary column $(15 \mathrm{~m} \times 0.1 \mathrm{~mm}$ i.d., $0.1 \mathrm{~mm}$ film thickness $)$, flame ionization detector and split/splitless injector. Samples were injected in splitless mode at an oven temperature of $120^{\circ} \mathrm{C}$, and after injection, the oven temperature was increased to $270{ }^{\circ} \mathrm{C}$ at $10{ }^{\circ} \mathrm{C} \min ^{-1}$ and then to $310{ }^{\circ} \mathrm{C}$ at $5{ }^{\circ} \mathrm{C} \mathrm{min}{ }^{-1}$. Peaks were quantified with Agilent Technologies ChemStation software (USA).

Confirmation of component identification was performed by GC-mass spectrometry (GC-MS) on a ThermoScientific 1310 GC coupled with a TSQ triple quadrupole. Samples were injected using a Tripleplus RSH autosampler into a non-polar HP-5 Ultra 2 bonded-phase column (50 $\mathrm{m} \times 0.32 \mathrm{~mm}$ i.d. $\times 0.17 \mu \mathrm{m}$ film thickness). The HP-5 column was of similar polarity to the column used for $\mathrm{GC}$ analyses. The initial oven temperature of $45^{\circ} \mathrm{C}$ was held for $1 \mathrm{~min}$, followed by temperature increase at $30^{\circ} \mathrm{C}$ per min to $140{ }^{\circ} \mathrm{C}$, then at $3{ }^{\circ} \mathrm{C}$ per min to $310^{\circ} \mathrm{C}$ which was held for $12 \mathrm{~min}$. Helium was used as the carrier gas. Mass spectrometer operating conditions were: electron impact energy $70 \mathrm{eV}$, emission current $250 \mu \mathrm{A}$, transfer line $310^{\circ} \mathrm{C}$, source temperature $240{ }^{\circ} \mathrm{C}$, scan rate $0.8 \mathrm{scan} \mathrm{s}^{-1}$ and mass range $40-$ 650 Da. Mass spectra were acquired and processed with Thermo Scientific XcaliburTM software (USA). Results are presented as the mean of area percent $(\%), \mathrm{mg} \mathrm{g}^{-1}$ biomass \pm standard deviation (SD) and as $\mathrm{mg} \mathrm{L}^{-1}$ of culture. All samples were prepared in triplicate.

\section{Determination of quality of the microbial oil for biodiesel production}

Qualitative properties of the samples for potential use as biodiesel were determined according to the fatty acid profiles obtained from cells grown to the exponential phase in MCBHB medium followed by assuming a winterization or other process (Table 4), leading to the removal of LC-PUFA. This mathematical analysis was carried out to investigate potential use of the oil by-product remaining after the purification of the thraustochytrid crude oil, primarily aimed at 
production of omega-3 for commercial application. The saponification value (SV), iodine value (IV) and cetane number (CN) were calculated using the following Eqs. (1), (2) and (3), respectively, as described in Francisco et al. (2010):

$$
\begin{aligned}
& \mathrm{SV}=\sum 560 N / M \\
& \mathrm{IV}=\sum 245 D N / M \\
& \mathrm{CN}=46.3+(5458 / \mathrm{SV})-(0.255 \times \mathrm{IV})
\end{aligned}
$$

where $N$ is the percentage of each fatty acid component, $D$ is the number of double bonds and $M$ is the molecular weight (amu) of the FAME. The molecular weights of FAME used were listed by Møller (2011).

The degree of unsaturation (DU), the long-chain saturation factor (LCSF) and the cold filter plugging point (CFPP) were estimated by Eqs. (4), (5) and (6) (Ramos et al. 2009):

$$
\begin{aligned}
& \mathrm{DU}=\text { MUFA }+(2 \times \text { PUFA }) \\
& \begin{aligned}
\mathrm{LCSF}= & (0.1 \times 16: 0)+(0.5 \times 18: 0)+(1 \times 20: 0) \\
& +(1.5 \times 22: 0)+(2 \times 24: 0)
\end{aligned} \\
& \mathrm{CFPP}\left({ }^{A} C\right)=(3.1417 \times \mathrm{LCSF})-16.477
\end{aligned}
$$

where MUFA and PUFA are the percentages of the monounsaturated fatty acids and polyunsaturated fatty acids, respectively, and 16:0, 18:0, 20:0, 22:0 and 24:0 are the percentage for these respective fatty acids.

\section{Results and discussion}

\section{Effect of growth phase and medium on fatty acid composition, final biomass and total fatty acid yields}

The growth phase very clearly impacted the fatty acid composition (expressed as \% of TFA) in both MCBHB and modified GYP media, as shown in Tables 1 and 2, respectively. In general, the relative levels of PUFA in both media increased during the stationary phase, while the proportion of saturated fatty acids (SFA) and monounsaturated fatty acids (MUFA) decreased. When grown in MCBHB medium, only strain SW7T8C showed a slight increase in the proportion of SFA and MUFA in the stationary phase, from 38.2 to $40.6 \%$ and 1.8 to $2.5 \%$ of TFA, respectively. In thraustochytrids, the biosynthetic pathways involved in the production of PUFA are the conventional fatty acid synthase route alongside that for polyketide synthase (PKS). The former is considered as the aerobic pathway and involves elongation of the carbon chain and formation of double bonds through a succession of elongase and desaturase enzymes (Ratledge 2004). In contrast, the PKS is sometimes described as the anaerobic pathway, and although not yet fully understood, it involves a "block building" system using the condensing enzyme 3ketoacyl synthase among others. In both cases, LC-PUFA synthesis uses SFA and MUFA as precursors (Metz et al. 2001). Therefore, whichever of these pathways is primarily used by these microorganisms, the synthesis of LC-PUFA as an end product over time will result in a decrease of the precursors, which was observed as lower relative levels of SFA and MUFA and higher levels of LC-PUFA in the stationary phase of growth. In addition, the increase of PUFA in the stationary phase of growth can be partially explained by the decreased total fatty acid yields in the same phase by almost all strains (Fig. 1). This can result in higher relative levels of PUFA due to lower oil content. However, this is not true for all strains, such as the strains SW7T7C and Sicyoidochytrium sp. which showed both higher total fatty acid yields and relative levels of PUFA in stationary phase.

Higher biomass concentrations (Fig. 2) and fatty acid yields (Fig. 1) were reached in MCBHB medium for all strains regardless of the growth phase, with the exception of Sicyoidochytrium sp. for which a higher biomass was observed in modified GYP at the late stationary phase of growth. However, in these screening media, the total lipid contents observed for the British isolates were low, ranging from 1.6 to $7.5 \%$ of dry cell weight regardless of the type of medium used. Hence, most of the fatty acids extracted are likely derived from membrane phospholipids rather than triacylglycerols. Similar results were obtained by Bowles et al. (1999), who showed that cold temperate strains (isolated from 59 to $61^{\circ} \mathrm{N}$ ) only accumulated lipids to between 2.0 and $7.1 \%$ of dry cell weight, while cool temperate isolates $\left(50-51^{\circ} \mathrm{N}\right)$ showed a lipid content between 1.6 and $13.6 \%$ of dry cell weight. Burja et al. (2006) also observed less than $10 \%$ of total lipid in dry cell weight biomass, for most of their isolates from cold Atlantic Canadian waters (from 40 to $50^{\circ} \mathrm{N}$ ). For industrial applications, the total fatty acid yields must be improved to be commercially viable for use in the food and aquaculture industries. However, a higher glucose concentration (2\% in modified GYP compared to $0.1 \%$ in MCBHB) did not induce a higher final biomass concentration, nor a higher accumulation of total lipids. This may be attributed either by the nutrient limitation in GYP (nitrogen or micronutrient insufficiency), or by an excess of glucose affecting growth in MCBHB.

Alternatively, the British thraustochytrids may be unable to utilize glucose and utilize another carbon source, such as amino acids. If this is the case, it implies that the higher biomass reached in MCBHB was due to other nutrients present such as peptones, yeast extract and brain heart broth that are complex nitrogen and carbon sources. Indeed, the total nitrogen and total carbon content in MCBHB were estimated to be 0.26 and $0.08 \%$ (ratio $\mathrm{C} / \mathrm{N}$ equal to 0.3 ), respectively, whereas for modified GYP the total nitrogen content was estimated to be $0.11 \%$ and $0.8 \%$ for total carbon (ratio $\mathrm{C} / \mathrm{N}$ equal to 7.45 ). Therefore, a higher nitrogen content and possibly a carbon 


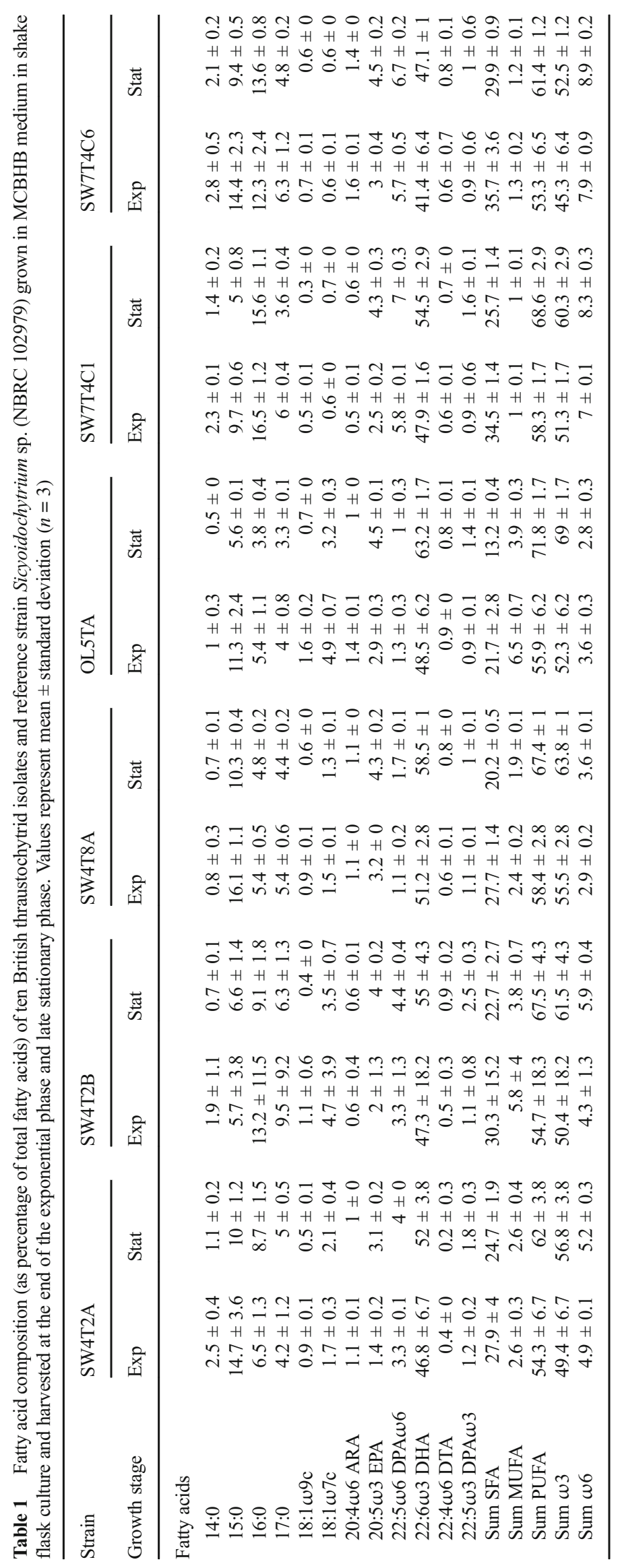

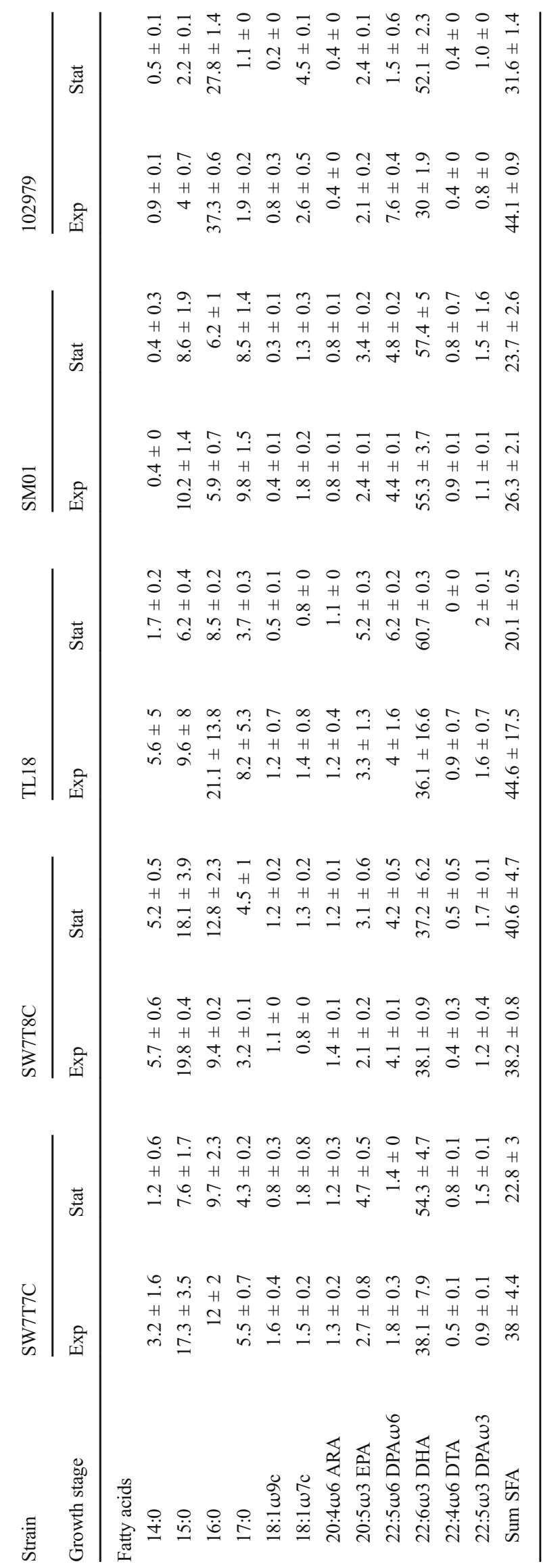


source derived from amino acids were more beneficial for thraustochytrids growth than a high concentration of glucose.

Among the British isolates, the highest fatty acid yields were achieved in MCBHB by strain SW7T8C (73 $\mathrm{mg} \mathrm{g}^{-1}$ DW) at the end of the exponential phase (Fig. 1), while the maximal biomass concentration at stationary phase of growth was found with strains OL5TA and SW7T4C6 (ca. $0.78 \mathrm{~g} \mathrm{~L}^{-1}$ ) (Fig. 2). Only strain TL18 showed higher fatty acid yield in modified GYP, which was achieved at the end of the exponential phase of growth (45.2 $\mathrm{mg} \mathrm{g}^{-1} \mathrm{DW}$ in modified GYP compared with $25.1 \mathrm{mg} \mathrm{g}^{-1} \mathrm{DW}$ in MCBHB) (Fig. 1). Hence, under the growth conditions used in this study, MCBHB was generally found to be a more suitable growth medium because higher fatty acid yields and biomass concentrations were achieved. However, we note that in terms of yield, none of the strains out-performed Sicyoidochytrium sp. NBRC 102979. Again, similar final biomass concentrations were found by Bowles et al. (1999) and Burja et al. (2006) for strains isolated from other cold water environments. In both studies, a medium containing $0.5 \%$ glucose was used, and final biomass concentrations achieved ranged between 0.1 and $1.5 \mathrm{~g} \mathrm{~L}^{-1}$ during the initial screening stage. Therefore, the performance of the British strains was similar to strains that had been isolated from cold Atlantic waters, with similar biomass and total fatty acid yields obtained at the screening stage. Since lipid accumulation does not appear to be enhanced by an increased glucose concentration, an approach to achieving more competitive biomass concentration and total lipid yields may require development of an optimized MCBHB medium containing different nutrients such as complex carbon and nitrogen sources. Therefore, higher yields are also likely to be achieved, possibly by changing other aspects of the medium and/or growth conditions.

\section{Effect of medium on PUFA profiles}

The total PUFA composition in the stationary phase across all the strains studied ranged from 47.9 to $75.1 \%$ of TFA in MCBHB medium (Table 1) and from 48.8 to $76.5 \%$ of TFA in modified GYP medium (Table 2). In MCBHB medium, the highest DHA levels of 63.2 and $60.7 \%$, respectively, of the TFA were produced by strains OL5TA and TL18, while maximum DHA levels in modified GYP medium were produced by strains SW7T8C, TL18 and OL5TA at 66, 67 and $60 \%$ of the TFA. Overall, the medium type had little effect on the PUFA profile of the strains in the stationary phase of growth (Fig. 3); the production of omega-3 fatty acids prevailed over the production of omega- 6 fatty acids in all cases, with DHA as the predominant PUFA component. The only exception was that a greater relative level of DPAw6 was observed for strains SW7T4C1 (10\% of PUFA) and SW7T4C6 (11\% of PUFA) in MCBHB medium (Fig. 3), with lower relative 


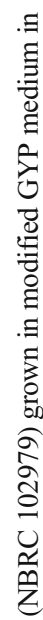

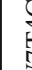

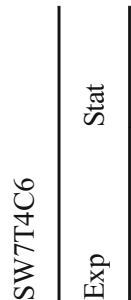

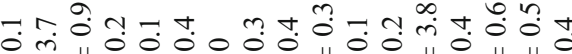

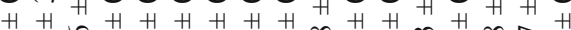

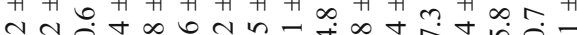

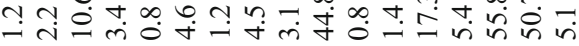

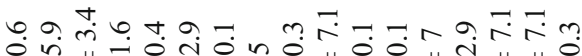

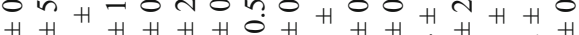

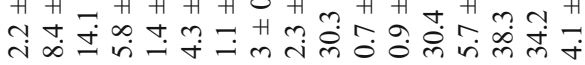

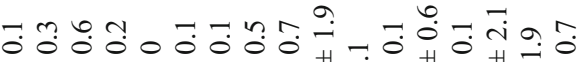

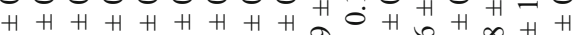

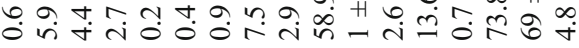

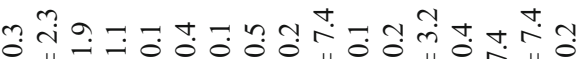
H

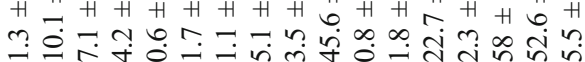

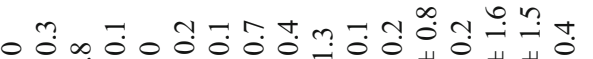

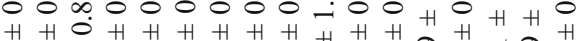

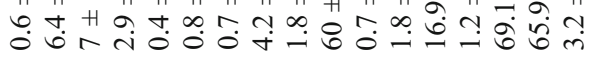

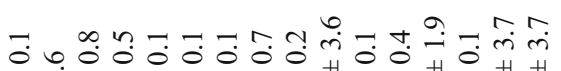
H- H H H H H H H H H H H H H 仓े

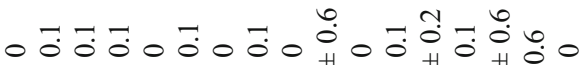
H H H H H H H H H H H H H H H H H H H H H H H H H H H H H ํㅜㅇ

m:்

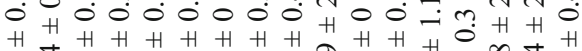
I. กุ $0 \dot{m}^{H} 00-10000$

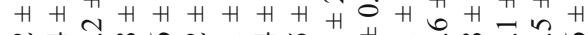

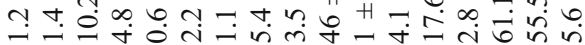

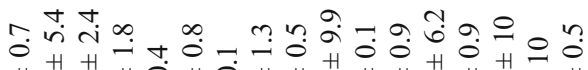

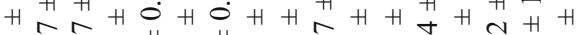

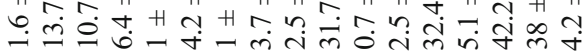

ت

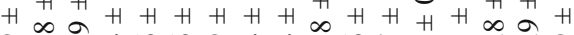

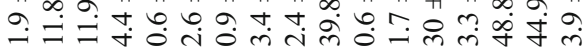

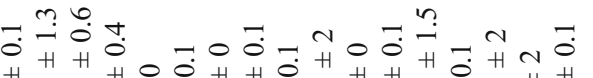

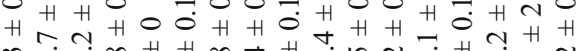
m드

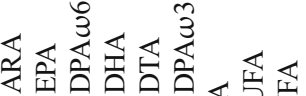

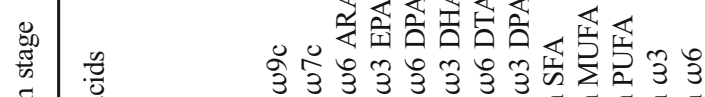

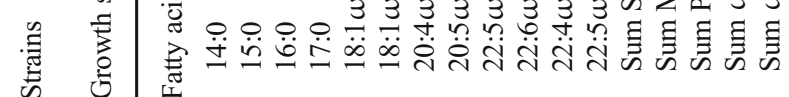

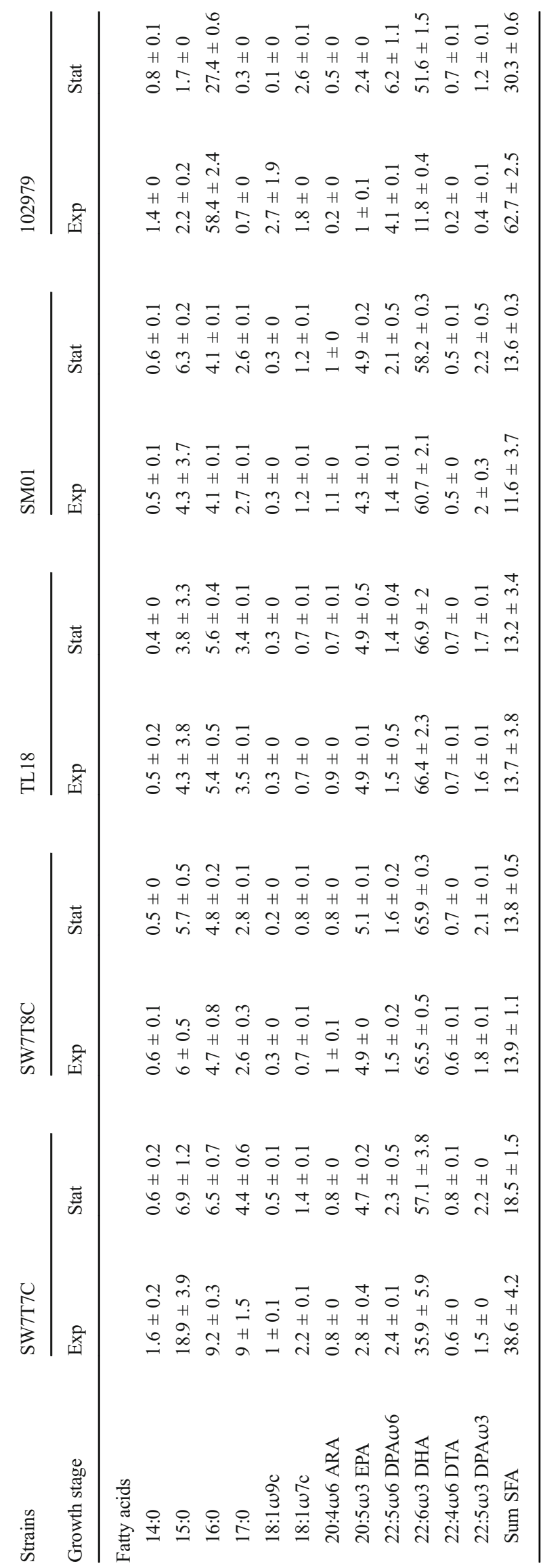




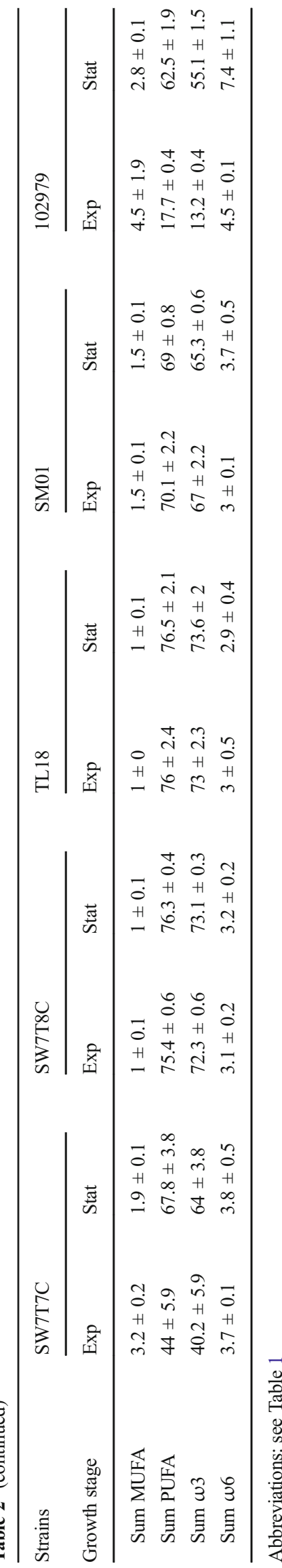

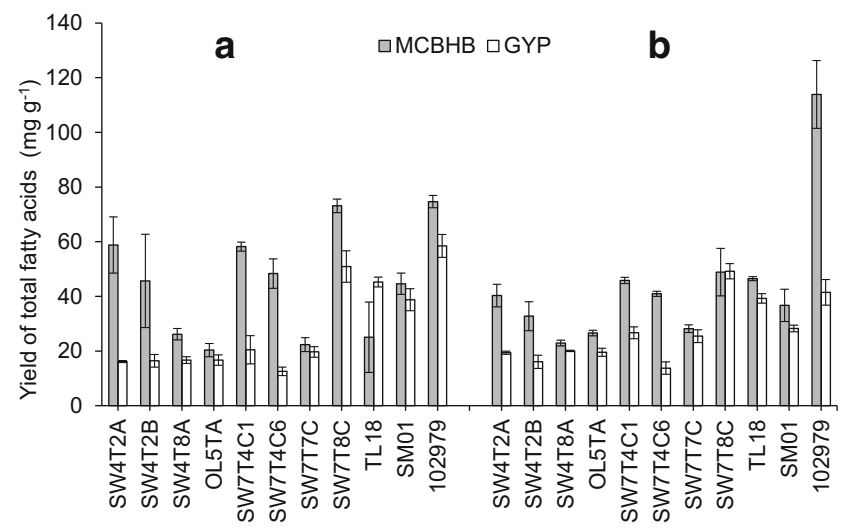

Fig. 1 Fatty acid yields ( $\mathrm{mg} \mathrm{g}^{-1}$ of dry cell weight) of ten British isolates and reference strain Sicyoidochytrium sp. (NBRC 102979) grown in MCBHB (grey bar) and modified GYP (open bar) media at the a end of the exponential phase and $\mathbf{b}$ late stationary phase. Values represent mean \pm standard deviation $(n=3)$

levels of DPAw6 produced in modified GYP (4 and 5\% of PUFA, respectively).

Thus, the fatty acid composition of the British isolates showed a comparable PUFA profile in modified GYP and in MCBHB media, with a high DHA level (average $83 \%$ of total PUFA) and low levels of EPA (average $7 \%$ of total PUFA) and DPA 66 (average 5\% of total PUFA). These results are in agreement with those of Huang et al. (2003) who observed no change in PUFA profiles of strains collected in coastal areas of Japan and Fiji when using different media containing different glucose concentrations. From these data, the British strains could potentially be of commercial interest for omega3 oil production, as the DHA level (expressed as \% TFA) is, to the best of our knowledge, among the highest recorded to date from an initial screening study. The highest level of DHA production by a thraustochytrid previously reported during a screening study was $61 \%$ of TFA by Aurantiochytrium sp. TC-39 (Lee Chang et al. 2012). As discussed below, further

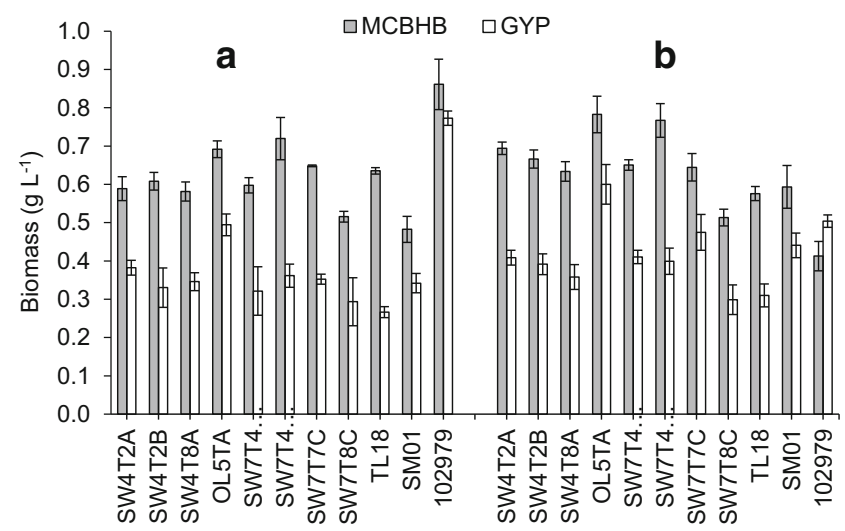

Fig. 2 Biomass concentration $\left(\mathrm{g} \mathrm{L}^{-1}\right)$ of ten British isolates and reference strain Sicyoidochytrium sp. (NBRC 102979) grown in MCBHB (grey bar) and modified GYP (open bar) media at the a end of the exponential phase and $\mathbf{b}$ late stationary phase. Values represent mean \pm standard deviation $(n=3)$ 


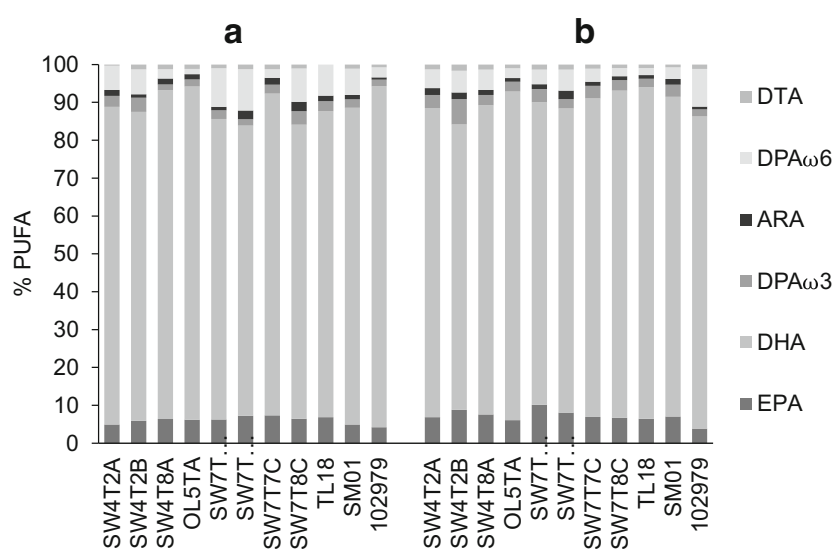

Fig. 3 PUFA profiles expressed as \% of total PUFA of ten British thraustochytrid isolates and reference strain Sicyoidochytrium sp. (NBRC 102979) grown in a MCBHB and $\mathbf{b}$ modified GYP medium in late stationary phase of growth. Values represent mean of independent triplicate $(n=3)$

work to optimize DHA yields is warranted in order to explore the biotechnological potential of these strains.

\section{DHA concentrations}

As described above, thraustochytrid strains grown in MCBHB medium showed higher fatty acid yields and biomass production compared to growth in modified GYP medium, while relative (\% TFA) levels of PUFA were higher in the stationary phase. Therefore, the concentration of DHA in milligram per liter in MCBHB medium at stationary phase was examined. Among the British isolates, strains SW7T4C1 and TL18 showed the highest production with DHA concentration reaching $16 \mathrm{mg} \mathrm{L}^{-1}$ on average (Fig. 4). These yields were lower than for the reference strain Sicyoidochytrium sp. NBRC 102979 (24.6 $\mathrm{mg} \mathrm{L}^{-1}$ ) and considerably lower than concentrations reported in other screening studies, with for instance Aurantiochytrium mangrovei FB3 which achieved a DHA concentration of $2.5 \mathrm{~g} \mathrm{~L}^{-1}$ (Fan et al. 2007). However, the low DHA concentrations achieved with the British strains can be mainly attributed to a low final biomass concentration reached in MCBHB. Indeed, when proportional levels of DHA were compared, the British strains exhibited the highest values (up to 63\% DHA of TFA in MCBHB) (Table 3). These observations suggest that in a more suitable medium, higher levels of biomass and DHA concentrations could be achieved. For instance, for the organism Aurantiochytrium sp. TC-20, Lee Chang et al. (2012) reported a similar low DHA concentration (40 $\left.\mathrm{mg} \mathrm{L}^{-1}\right)$, final biomass concentration $\left(1 \mathrm{~g} \mathrm{~L}^{-1}\right)$, total fatty acid content $(7.8 \%)$ and a relatively high level of DHA (52\% of TFA) during initial screening of this strain. Nonetheless, in subsequent studies with the same organism using further optimized culture conditions (fed-batch bioreactor with glycerol and varying nutritional supplements), the biomass reached $71 \mathrm{~g} \mathrm{~L}^{-1}$ and the DHA concentration was improved to $14 \mathrm{~g} \mathrm{~L}^{-1}$ of culture medium, while the relative proportion of DHA decreased slightly to $39 \%$ of TFA (Lee Chang et al. 2013a). Thus, it was demonstrated that it was possible to greatly increase the final biomass by optimizing culture conditions and culture strategy, while having only a moderate effect on the relative levels of DHA, hence allowing for considerable improvement in the culture DHA concentration. Taken collectively, the British strains show considerable potential for achieving higher

Table 3 Comparison of relative levels (as \% TFA) and culture concentrations of DHA at the screening stage for different thraustochytrid strains

\begin{tabular}{|c|c|c|c|c|c|c|c|}
\hline Strain & Location & Climate & $\begin{array}{l}\text { DHA (\% of } \\
\text { TFA) }\end{array}$ & $\begin{array}{l}\text { Fatty acids } \\
\text { yield } \\
\left(\mathrm{mg} \mathrm{g}^{-1}\right)\end{array}$ & $\begin{array}{l}\text { Biomass } \\
\left(\mathrm{g} \mathrm{L}^{-1}\right)\end{array}$ & $\begin{array}{l}\text { DHA } \\
\text { concentration } \\
\left(\mathrm{mg} \mathrm{L}^{-1}\right)\end{array}$ & Reference \\
\hline Aurantiochytrium TC-20 & $\begin{array}{l}\text { North Queensland, } \\
\text { Australia }\end{array}$ & Tropical & 51.7 & 78 & 1 & 40 & $\begin{array}{l}\text { Lee Chang et al. } \\
\text { (2012) }\end{array}$ \\
\hline 4-P-J & Hong Kong, China & Sub-tropical & 31.0 & - & 15.6 & - & Li et al. (2009) \\
\hline $\begin{array}{r}\text { Aurantiochytrium } \\
\text { mangrovei } \mathrm{FB} 3\end{array}$ & Hong Kong, China & Sub-tropical & 29.6 & 680.4 & 12.2 & 2457 & Fan et al. (2007) \\
\hline $\begin{array}{c}\text { Sicyoidochytrium sp. } \\
\text { NBRC } 102979\end{array}$ & Iriomote Island, Japan & Sub-tropical & 52.1 & 113.9 & 0.41 & 24 & This study \\
\hline Aurantiochytrium TC-39 & South East, Tasmania & $\begin{array}{l}\text { Cool } \\
\text { temperate }\end{array}$ & 60.8 & 57 & 1.3 & 45 & $\begin{array}{l}\text { Lee Chang et al. } \\
\text { (2012) }\end{array}$ \\
\hline TL18 & North Sea, Scotland & $\begin{array}{l}\text { Cold } \\
\text { temperate }\end{array}$ & 60.7 & 46.5 & 0.58 & 16 & This study \\
\hline SW7T4C1 & North Sea, Scotland & $\begin{array}{l}\text { Cold } \\
\text { temperate }\end{array}$ & 54.5 & 45.8 & 0.65 & 16 & This study \\
\hline OL5TA & North Sea, Scotland & $\begin{array}{l}\text { Cold } \\
\text { temperate }\end{array}$ & 63.2 & 26.6 & 0.78 & 13 & This study \\
\hline
\end{tabular}




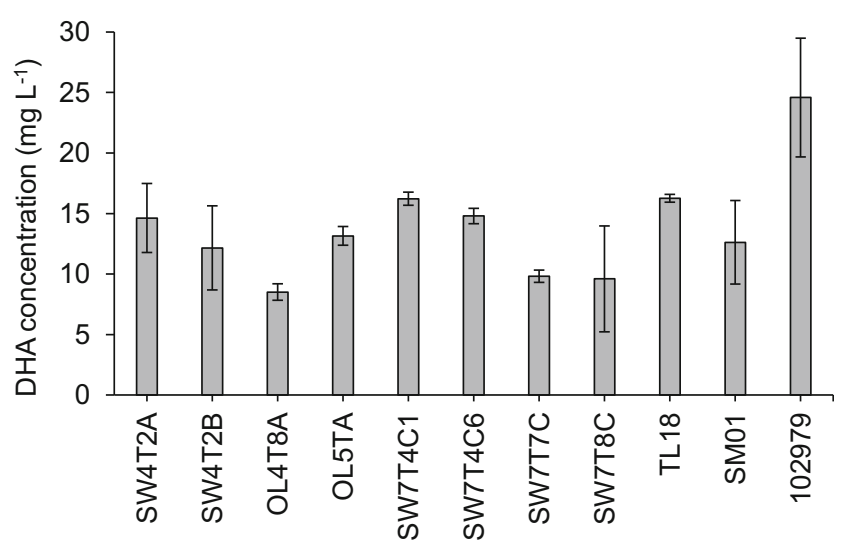

Fig. 4 DHA content $\left(\mathrm{mg} \mathrm{L}^{-1}\right)$ of ten British isolates and reference strain Sicyoidochytrium sp. (NRBC 102979) grown in MCBHB medium in late stationary phase of growth. Values represent mean \pm standard deviation $(n=3)$

DHA concentrations, due to their high relative levels of DHA coupled with the scope for improvement in final biomass concentrations and thereby fatty acid yields.

\section{Mathematical model to assess the by-product oil for use as biodiesel}

To maximize the possible future industrial potential of the British thraustochytrid strains, a post-fermentation winterization or other separation/fractionation step was assumed. Briefly, the winterization (or other) process is a physical process that enables the separation of oils (generally composed of LC-PUFA) from fats (generally composed of SFA) according to their melting point by means of cooling and filtration.
During the cooling step, SFA, which have higher melting points than LC-PUFA, will solidify first (stearin) while LCPUFA will remain liquid (olein), thus allowing their separation and purification by subsequent filtration. Therefore, this process has the specific objective to increase the content of omega-3, and thus DHA, of the primary oil by removing SFA and MUFA. Meanwhile, this could potentially also enable the coproduction of microbial biodiesel from the residual stearin (Lee Chang et al. 2013b). To assess this, relative levels of SFA and MUFA from cultures grown in MCBHB medium and harvested at the end of the exponential phase were recalculated assuming that LC-PUFA were removed from the lipid phase by winterization, leaving MUFA and SFA as the predominant fatty acids (Table 4). Indeed, shorter chain (SC, $\leq \mathrm{C}_{18}$ ) fatty acids and a high degree of saturation are often seen as more suitable for optimal biodiesel properties (Lee Chang et al. 2013b).

The qualitative properties of the thraustochytrid residual oil for potential biodiesel application, based on the fatty acids profiles after winterization, are reported in Table 5. The saponification values ranged from 109 to 183 , showing disparity of the average fatty acid chain length of the British strains. The iodine values ranged from 3 to 21 , showing different oxidative stability profiles. For instance, the high iodine value of strain OL5TA (21) and SW4T2B (18) could be explained by the high level of MUFA (23.6 and $20.2 \%$ of TFA, respectively), particularly $18: 1 \omega 7 \mathrm{c}$, thus reflecting a richness in double bonds of the oil. On the other hand, SW7T4C1 and SW7T4C6 showed a lower iodine value (3 and 6, respectively) due to their lower levels of $18: 1 \omega 7 \mathrm{c}(1.4$ and $1.3 \%$ of TFA, respectively) and high levels of SFA ( 88.9 and $84.8 \%$ of TFA,

Table 4 Theoretical fatty acid profile of oil derived from British thraustochytrids cultured in MCBHB medium and harvested at the exponential phase of growth followed by a theoretical winterization process. Values represent mean \pm standard deviation $(n=3)$

\begin{tabular}{lrrrrrrrrrrr}
\hline Strains & SW4T2A & SW4T2B & SW4T8A & OL5TA & SW7T4C1 & SW7T4C6 & SW7T7C & SW7T8C & TL18 & SM01 & 102979 \\
\hline $13: 0$ & $3.3 \pm 0.2$ & $0.3 \pm 1.6$ & $0 \pm 1.7$ & $0.1 \pm 1.7$ & $1.1 \pm 0.2$ & $2.5 \pm 0.2$ & $1.2 \pm 1.7$ & $7.1 \pm 0.9$ & $0.7 \pm 1.2$ & $0 \pm 1.7$ & $0 \pm 0$ \\
$14: 0$ & $5.9 \pm 0.2$ & $4.3 \pm 0.7$ & $2 \pm 0.4$ & $2.5 \pm 0.3$ & $5.7 \pm 0.1$ & $6.2 \pm 0.2$ & $6.2 \pm 0.5$ & $11.5 \pm 0.1$ & $10.8 \pm 1$ & $1 \pm 0.1$ & $1.5 \pm 0.1$ \\
$15: 0$ & $35.4 \pm 0.3$ & $12.9 \pm 0.7$ & $41.3 \pm 0.1$ & $27.7 \pm 0.2$ & $23.9 \pm 0.1$ & $32.5 \pm 0.2$ & $33.5 \pm 0.3$ & $39.8 \pm 0$ & $18.6 \pm 0.9$ & $29.7 \pm 0.1$ & $6.9 \pm 0.2$ \\
$16: 1 \omega 9 \mathrm{c}$ & $0.1 \pm 0.9$ & $0.2 \pm 1.2$ & $1.4 \pm 0.1$ & $1.2 \pm 1.2$ & $0 \pm 0$ & $0.1 \pm 0.9$ & $0.3 \pm 0.3$ & $0.1 \pm 1.7$ & $0 \pm 0$ & $0 \pm 0$ & $0 \pm 1.7$ \\
$16: 1 \omega 7 \mathrm{c}$ & $3.5 \pm 0.2$ & $1.9 \pm 0.7$ & $0.1 \pm 0.9$ & $1.6 \pm 0.9$ & $0.4 \pm 0.2$ & $1.2 \pm 0.1$ & $2.3 \pm 0.3$ & $2.3 \pm 0$ & $1.8 \pm 0.7$ & $0.6 \pm 0.1$ & $3.3 \pm 0.2$ \\
$16: 0$ & $15.6 \pm 0.2$ & $30.1 \pm 0.9$ & $13.8 \pm 0.1$ & $13.1 \pm 0.2$ & $40.6 \pm 0.1$ & $27.9 \pm 0.2$ & $23.3 \pm 0.2$ & $19 \pm 0$ & $40.7 \pm 0.7$ & $17.2 \pm 0.1$ & $64 \pm 0$ \\
$17: 1 \omega 8 \mathrm{c}$ & $2.1 \pm 0.2$ & $1.7 \pm 0.7$ & $1 \pm 0.1$ & $1.9 \pm 0.2$ & $0.3 \pm 0.2$ & $1.1 \pm 0.3$ & $1.3 \pm 0.4$ & $2.3 \pm 0.1$ & $1 \pm 1$ & $1.2 \pm 0.2$ & $0.7 \pm 0.3$ \\
$17: 1 \omega 6 \mathrm{c}$ & $2.6 \pm 0.2$ & $2.6 \pm 0.8$ & $1.3 \pm 0.1$ & $2.7 \pm 0.2$ & $0.1 \pm 0.9$ & $0.7 \pm 0.3$ & $0.9 \pm 0.2$ & $1.1 \pm 0.2$ & $0.6 \pm 1$ & $2 \pm 0.3$ & $0.1 \pm 0.9$ \\
$17: 0$ & $10.1 \pm 0.3$ & $21.8 \pm 1$ & $13.9 \pm 0.1$ & $9.7 \pm 0.2$ & $14.9 \pm 0.1$ & $14.2 \pm 0.2$ & $10.7 \pm 0.2$ & $6.4 \pm 0$ & $15.9 \pm 0.7$ & $28.5 \pm 0.2$ & $3.2 \pm 0.1$ \\
$18: 1 \omega 9 \mathrm{c}$ & $2 \pm 0.1$ & $2.4 \pm 0.7$ & $2.2 \pm 0.1$ & $3.9 \pm 0.2$ & $1.1 \pm 0.1$ & $1.7 \pm 0.2$ & $3.1 \pm 0.3$ & $2.1 \pm 0$ & $2.3 \pm 0.7$ & $1 \pm 0.2$ & $1.4 \pm 0.4$ \\
$18: 1 \omega 7 \mathrm{c}$ & $4.1 \pm 0.2$ & $10.8 \pm 0.9$ & $3.8 \pm 0.1$ & $11.9 \pm 0.2$ & $1.4 \pm 0.1$ & $1.3 \pm 0.2$ & $2.8 \pm 0.2$ & $1.6 \pm 0$ & $2.7 \pm 0.7$ & $5.2 \pm 0.1$ & $4.5 \pm 0.2$ \\
$18: 0$ & $0.9 \pm 0.2$ & $1.6 \pm 0.9$ & $0.8 \pm 0.1$ & $0.9 \pm 0.2$ & $2.6 \pm 0.1$ & $1.4 \pm 0.2$ & $1.4 \pm 0.2$ & $1 \pm 0.1$ & $2 \pm 0.7$ & $0.8 \pm 0.1$ & $0.8 \pm 0.1$ \\
$19: 1 \mathrm{a}$ & $0.7 \pm 0.2$ & $0.5 \pm 0.6$ & $0.3 \pm 0.1$ & $0.5 \pm 0.1$ & $0 \pm 0$ & $0.5 \pm 0.3$ & $0.6 \pm 0.4$ & $1 \pm 0.1$ & $0.3 \pm 0.9$ & $0.3 \pm 0.9$ & $0 \pm 0.9$ \\
Sum SFA & $71.3 \pm 0.2$ & $71 \pm 0.6$ & $71.9 \pm 0.1$ & $54.1 \pm 0.2$ & $88.9 \pm 0$ & $84.8 \pm 0.2$ & $76.3 \pm 0.2$ & $84.8 \pm 0.1$ & $88.8 \pm 0.5$ & $77.3 \pm 0.1$ & $76.4 \pm 0.1$ \\
Sum MUFA & $15.2 \pm 0.1$ & $20.2 \pm 0.6$ & $10.2 \pm 0.1$ & $23.6 \pm 0.2$ & $3.4 \pm 0.1$ & $6.6 \pm 0.1$ & $11.4 \pm 0.2$ & $10.6 \pm 0$ & $8.7 \pm 0.5$ & $10.2 \pm 0.1$ & $10 \pm 0.1$ \\
\hline
\end{tabular}


Table 5 Qualitative properties of biodiesel for ten British thraustochytrid strains and reference strain Sicyoidochytrium sp. based on their fatty acid profiles in Table 4

\begin{tabular}{lcccccccrrrr}
\hline Strain & SW4T2A & SW4T2B & SW4T8A & OL5TA & SW7T4C1 & SW7T4C6 & SW7T7C & SW7T8C & TL18 & SM01 & 102979 \\
\hline SV & 183 & 114 & 131 & 109 & 154 & 156 & 149 & 182 & 159 & 111 & 159 \\
IV & 14 & 18 & 9 & 21 & 3 & 6 & 10 & 9 & 8 & 9 & 9 \\
CN & 73 & 90 & 86 & 91 & 81 & 80 & 81 & 74 & 79 & 93 & 78 \\
DU & 30 & 39 & 20 & 46 & 6 & 13 & 22 & 21 & 17 & 20 & 20 \\
LCSF & 2.0 & 3.8 & 1.8 & 1.8 & 5.4 & 3.5 & 3.0 & 2.4 & 5.1 & 2.1 & 6.8 \\
CFPP & -10.1 & -4.5 & -10.8 & -10.9 & 0.4 & -5.5 & -6.9 & -9.0 & -0.5 & -9.8 & 4.9 \\
\hline
\end{tabular}

$S V$ saponification value is correlated with the average chain length (molecular weight) of the fatty acids profile, $I V$ the iodine value represents the oxidative stability by evaluating the total unsaturation of the fatty acid profile, $C N$ the cetane number represents the ignition property, $D U$ denotes the degree of unsaturation of the fatty acids which represents the oxidative stability during long term storage, LCSF a high long-chain saturation factor indicates an oil rich in LC-SFA and therefore more sensitive to crystallization, $C F P P\left({ }^{\circ} \mathrm{C}\right)$ the cold filter plugging point is the temperature at which the biodiesel tends to crystallize which can clog the filters and fuel lines (Ruangsomboon 2015)

respectively). This feature is confirmed by the value for the degree of unsaturation, which is directly proportional to the relative level of unsaturated fatty acids. Good ignition properties are achieved with a fatty acid profile rich in SC-SFA, which implies low iodine and saponification values and therefore a high cetane number. The strains SW4T2B, OL5TA and SM01 exhibited the highest cetane numbers $(90,91$ and 93 , respectively). Nonetheless, the remaining strains still showed a high cetane number ranging from 73 to 86 . The LCSF is a good indicator of the richness of an oil in LC-SFA, which are undesirable for biodiesel as they have high melting points. Indeed, for biodiesel application, the oil must remain liquid at low temperatures to avoid clogging of engines and fuel lines. Thus, a low LCSF value will result in a low CFPP, which is a good intrinsic property for biodiesel. In the present study, strains SW4T2A, SW4T8A, OL5TA and SM01 showed very low CFPP values of $-9.8{ }^{\circ} \mathrm{C}$ or below, while strains SW7T4C1 and TL18 showed the highest CFPP of 0.4 and $-0.5^{\circ} \mathrm{C}$, respectively.

As shown in Table 6, British thraustochytrids under the growth conditions used in this study, and after a winterization process, could meet the reference standard criteria for biodiesel application according to the European Standard EN 14214 and ASTM International standard D6751. All of their cetane numbers are above 51 and iodine values below 120, mainly due to their high SFA composition. In comparison, Byreddy et al. (2015), in a similar sub-study on biodiesel, found that full crude oil extracted from Schizochytrium sp. S31 and Thraustochytrium sp. AMCQS5-5 did not meet the biodiesel standard requirement due to the high relative level of LCPUFA contained in both strains. Nonetheless, Schizochytrium sp. S056 almost met the EN 14214 standard (Chen et al. 2015), showing that thraustochytrid crude oil rich in palmitic acid could potentially be used for biodiesel

Table 6 Intrinsic properties calculated from fatty acid profiles of oils from thraustochytrids and other sources for potential biodiesel application

\begin{tabular}{lccccccl}
\hline & CN & IV & SV & CFPP & DU & LCSF & References \\
\hline EN 14214 & $>51$ & $<120$ & & - & - & - & \\
ASTM D6751 & $>47$ & - & & - & - & - & \\
Schizochytrium sp. S056 & 49 & 118 & & 7.5 & & & Chen et al. (2015) \\
Schizochytrium sp. S31 & 47 & 99 & 234 & 4.5 & 63 & 3.8 & Byreddy et al. (2015) \\
Thraustochytrium sp. AMCQS5-5 & 44 & 157 & 165 & 1.9 & 70 & 5.8 & Byreddy et al. (2015) \\
British thraustochytrid OL5TA & 91 & 21 & 109 & -10.9 & 46 & 1.8 & This study \\
British thraustochytrid SM01 & 93 & 9 & 111 & -9.8 & 20 & 2.1 & This study \\
Chlorella & 57 & 65 & 225 & -4.6 & 71 & 3.8 & Francisco et al. (2010) \\
Phaeodactylum & 56 & 59 & 217 & -12.3 & 53 & 1.3 & Francisco et al. (2010) \\
Sunflower & 50 & 132 & $(193)$ & -3 & - & - & Ramos et al. (2009) (Gopinath et al. 2009) \\
Palm & 61 & 57 & $(205)$ & 10 & - & - & Ramos et al. (2009) (Gopinath et al. 2009) \\
Microcystis aeruginosa NPCD-1 & - & 57 & 210 & & 61 & 5.7 & Da Rós et al. (2013) \\
Trichormus sp.CENA77 & - & 68 & 213 & & 70 & 5 & Da Rós et al. (2013) \\
\hline
\end{tabular}

Abbreviations: see Table 5 
exploitation directly without requiring a winterization step. However, the CFPP $\left(7.5^{\circ} \mathrm{C}\right)$ was higher when compared to the findings of this study (as low as $-10.9^{\circ} \mathrm{C}$ ) which would be a disadvantage (Table 6). Crude oil from other microorganisms, such as phototrophic microalgae and cyanobacteria, also have shown potential for biodiesel application with low CFPP values, but higher SV and DU values were observed, suggesting higher content in LC-PUFA, thus showing the importance of the winterization step to remove these (Francisco et al. 2010; Da Rós et al. 2013). Lee Chang et al. (2015) showed that thraustochytrid cultivation for hydroprocessed biodiesel production could be viable using agro-industrial by-products (e.g. molasses or glycerol) as sole carbon source. However, transesterified biodiesel had a low-energy return on energy invested factor of 0.43 (meaning that for 1 unit of energy produced, 2.32 units of energy are needed). As a result, additional research would still be required to improve growth conditions, fatty acid profile and yield for biodiesel production from thraustochytrids. The most promising way forward may rather see a primary objective of optimizing PUFA production by thraustochytrids with by-product oil lacking PUFA also being available for biodiesel application.

\section{Conclusion}

This initial screening study of new British thraustochytrid strains, based on their fatty acid profiles following growth in low-glucose and complex nitrogen source-rich medium MCBHB, showed that they have potential for DHA production, particularly strains TL18 and SW7T4C1 (highest culture DHA concentration) and strain OL5TA (highest relative proportion of TFA as DHA). Further work is now required to optimize culture conditions (including further investigation of different carbon and nitrogen sources in the medium) to increase the biomass and fatty acid yields of these British strains in order to generate competitive levels of production. In addition, a mathematical analysis of residual fatty acids after a theoretical winterization step revealed that the byproduct oil could be potentially suitable for biodiesel production.

Acknowledgements This work was supported by a James-Watt Scholarship to Loris Fossier and a personal donation from David J Alderman with grants from the Gen Foundation (UK registered charity number 1071026) and the Heriot Watt Annual Alumni Fund (Scottish registered Charity number SC000278) covering travel and accommodation expenses to the CSIRO where the fatty acid analyses were performed. We also thank Kirsty Fraser for permission to use isolate SM01 and D. J. Alderman for his helpful comments in the preparation of the manuscript.

Open Access This article is distributed under the terms of the Creative Commons Attribution 4.0 International License (http:// creativecommons.org/licenses/by/4.0/), which permits unrestricted use, distribution, and reproduction in any medium, provided you give appropriate credit to the original author(s) and the source, provide a link to the Creative Commons license, and indicate if changes were made.

\section{References}

Barclay WR, Meager KM, Abril JR (1994) Heterotrophic production of long chain omega-3 fatty acids utilizing algae and algae-like microorganisms. J Appl Phycol 6:123-129

Bowles RD, Hunt AE, Bremer GB, Duchars MG, Eaton RA (1999) Long-chain n-3 polyunsaturated fatty acid production by members of the marine protistan group the thraustochytrids: screening of isolates and optimization of docosahexaenoic acid production. $\mathrm{J}$ Biotechnol 70:193-202

Burja AM, Radianingtyas H, Windust A, Barrow CJ (2006) Isolation and characterization of polyunsaturated fatty acid producing Thraustochytrium species: screening of strains and optimization of omega-3 production. Appl Microbiol Biotechnol 72:1161-1169

Byreddy AR, Gupta A, Barrow CJ, Puri M (2015) Comparison of cell disruption methods for improving lipid extraction from thraustochytrid strains. Mar Drugs 13:5111-5127

Chen W, Ma L, Zhou P, Zhu Y, Wang X, Luo X, Bao Z, Yu L (2015) A novel feedstock for biodiesel production: the application of palmitic acid from Schizochytrium. Energy 86:128-138

Colomer R, Moreno-Nogueira JM, García-Luna PP, García-Peris P, García-de-Lorenzo A, Zarazaga A, Quecedo L, del Llano J, Usán L, Casimiro C (2007) N-3 fatty acids, cancer and cachexia: a systematic review of the literature. Br J Nutr 97:823-831

Da Rós PCM, Silva CSP, Silva-Stenico ME, Fiore MF, De Castro HF (2013) Assessment of chemical and physico-chemical properties of cyanobacterial lipids for biodiesel production. Mar Drugs 11:23652381

Energy Policy Act (2005) Pub. L. No. 109-58, 119 Stat. 459-497

European Commission (2003) Directive 2003/30/EC on the promotion of the use of biofuels or other renewable fuels for transport. 123:42-46

Fan KW, Vrijmoed LLP, Jones EBG (2002) Physiological studies of subtropical mangrove thraustochytrids. Bot Mar 45:50-57

Fan KW, Jiang Y, Faan YW, Chen F (2007) Lipid characterization of mangrove thraustochytrid-Schizochytrium mangrovei. J Agric Food Chem 55:2906-2910

FAO (2014) The state of world fisheries and aquaculture 2014. Food and Agriculture Organisation, Rome 223 pp

Francisco EC, Neves DB, Jacob-Lopes E, Franco TT (2010) Microalgae as feedstock for biodiesel production: carbon dioxide sequestration, lipid production and biofuel quality. J Chem Technol Biotechnol 85: 395-403

Gopinath A, Puhan S, Nagarajan G (2009) Theoretical modeling of iodine value and saponification value of biodiesel fuels from their fatty acid composition. Renew Energy 34:1806-1811

Honda D, Yokochi T, Nakahara T, Erata M, Higashihara T (1998) Schizochytrium limacinum sp. nov., a new thraustochytrid from a mangrove area in the west Pacific Ocean. Mycol Res 102:439-448

Horrocks LA, Yeo YK (1999) Health benefits of docosahexaenoic acid (DHA). Pharmacol Res 40:211-225

Huang J, Aki T, Yokochi T, Nakahara T, Honda D, Kawamoto S, Shigeta S, Ono K, Suzuki O (2003) Grouping newly isolated docosahexaenoic acid-producing thraustochytrids based on their polyunsaturated fatty acid profiles and comparative analysis of 18S rRNA genes. Mar Biotechnol 5:450-457

Lee Chang KJ, Mansour MP, Dunstan GA, Blackburn SI, Koutoulis A, Nichols PD (2011) Odd-chain polyunsaturated fatty acids in thraustochytrids. Phytochemistry 72:1460-1465 
Lee Chang KJ, Dunstan GA, Abell GCJ, Clementson LA, Blackburn SI, Nichols PD, Koutoulis A (2012) Biodiscovery of new Australian thraustochytrids for production of biodiesel and long-chain omega-3 oils. Appl Microbiol Biotechnol 93:2215-2231

Lee Chang KJ, Dumsday G, Nichols PD, Dunstan GA, Blackburn SI, Koutoulis A (2013a) High cell density cultivation of a novel Aurantiochytrium sp. strain TC 20 in a fed-batch system using glycerol to produce feedstock for biodiesel and omega-3 oils. Appl Microbiol Biotechnol 97:6907-6918

Lee Chang KJ, Nichols PD, Blackburn SI (2013b) More than biofuelspotential uses of microalgae as sources of high-value lipids. Lipid Technol 25:199-203

Lee Chang KJ, Nichols CM, Blackburn SI, Dunstan GA, Koutoulis A, Nichols PD (2014) Comparison of thraustochytrids Aurantiochytrium sp., Schizochytrium sp., Thraustochytrium sp., and Ulkenia sp. for production of biodiesel, long-chain omega-3 oils, and exopolysaccharide. Mar Biotechnol 16:396-411

Lee Chang KJ, Rye L, Dunstan GA, Grant T, Koutoulis A, Nichols PD, Blackburn SI (2015) Life cycle assessment: heterotrophic cultivation of thraustochytrids for biodiesel production. J Appl Phycol 27: 639-647

Lewis TE, Nichols PD, McMeekin TA (1999) The biotechnological potential of thraustochytrids. Mar Biotechnol 1:580-587

Li Q, Chen GQ, Fan KW, Lu FP, Aki T, Jiang Y (2009) Screening and characterization of squalene-producing thraustochytrids from Hong Kong mangroves. J Agric Food Chem 57:4267-4272

Manikan V, Nazir MYM, Kalil MS, Isa MHM, Kader AJA, Yusoff WMW, Hamid AA (2015) A new strain of docosahexaenoic acid producing microalga from Malaysian coastal waters. Algal Res 9: $40-47$

Meng X, Yang J, Xu X, Zhang L, Nie Q, Xian M (2009) Biodiesel production from oleaginous microorganisms. Renew Energy 34:1-5

Metz JG, Roessler P, Facciotti D, Levering C, Dittrich F, Lassner M, Valentine R, Lardizabal K, Domergue F, Yamada A, Yazawa K,
Knauf V, Browse J (2001) Production of polyunsaturated fatty acids by polyketide synthases in both prokaryotes and eukaryotes. Science 293:290-293

Møller A (2011) Fatty acid molecular weights. Danish Food Inf 1-7

Pike IH, Jackson A (2010) Fish oil: production and use now and in the future. Lipid Technol 22:59-61

Polglase JL (1980) A preliminary-report on the thraustochytrid(s) and labyrinthulid(s) associated with a pathological condition in the lesser octopus Eledone cirrhosa. Bot Mar 23:699-706

Polglase JL (1981) Thraustochytrids as potential pathogens of marine animals. Bull Br Mycological Soc 16:5

Ramos MJ, Fernández CM, Casas A, Rodríguez L, Pérez A (2009) Influence of fatty acid composition of raw materials on biodiesel properties. Bioresour Technol 100:261-268

Ratledge C (2004) Fatty acid biosynthesis in microorganisms being used for single cell oil production. Biochimie 86:807-815

Ratledge C (2012) Omega-3 biotechnology: errors and omissions. Biotechnol Adv 30:1746-1747

Rosa SM, Galvagno MA, Vélez CG (2011) Adjusting culture conditions to isolate thraustochytrids from temperate and cold environments in southern Argentina. Mycoscience 52:242-252

Ruangsomboon S (2015) Effects of different media and nitrogen sources and levels on growth and lipid of green microalga Botryococcus braunii KMITL and its biodiesel properties based on fatty acid composition. Bioresour Technol 191:377-384

Sargent J, Bell G, McEvoy L, Tocher D, Estevez A (1999) Recent developments in the essential fatty acid nutrition of fish. Aquaculture 177: 191-199

Swanson D, Block R, Mousa S (2012) Omega-3 fatty acids EPA and DHA: health benefits throughout life. Adv Nutr An Int Rev J 3:1-7

Tietenberg T, Lewis L (2009) Environmental and natural resource economics, 9th edn. Addison-Wesley, New Jersey 